\title{
Advances in Pharmacologic Stress Agents: Focus on Regadenoson
}

\author{
Sara G. Johnson ${ }^{1}$ and Scott Peters ${ }^{2}$ \\ ${ }^{I}$ Nuclear Medicine Service, VA San Diego Healthcare System, San Diego, California; and ${ }^{2}$ Astellas Pharma US, Inc., \\ Critical Care/Hospital Products (Cardiovascular), Deerfield, Illinois
}

Myocardial perfusion imaging using radionuclides is a wellestablished protocol for determining the diagnosis, prognosis, and management of coronary artery disease. Pharmacologic stress agents are used to induce cardiac hyperemia in patients unable to achieve the target workload by physical exercise alone. The vasodilators adenosine and dipyridamole are most commonly used, with dobutamine used only when these agents are contraindicated. However, because of frequent and intense side effects, as well as complex procedures both for patients and the nuclear medicine staff, there is room for improvement in these traditional stress agents. An ideal stress agent would be effective, safe, and well tolerated; have a simple protocol; be suitable for use in patients with reactive airway disease; and have few restrictions for the patient to adhere to before the procedure. Neither adenosine nor dipyridamole are receptorspecific, and act on $A_{1}, A_{2 A}, A_{2 B}$, and $A_{3}$ adenosine receptors. As it is the $A_{2 A}$ receptor that mediates the desired coronary vasodilation, the $A_{1}, A_{2 B}$, and $A_{3}$ adenosine receptors are deemed responsible for most side effects associated with adenosine and dipyridamole. $\mathrm{A}_{2 \mathrm{~A}}$-selective pharmacologic stress agents should mediate the required hyperemic response while reducing the frequency of adverse events. The only selective $A_{2 A}$ adenosine receptor agonist currently approved for clinical use as a pharmacologic stress agent for myocardial perfusion imaging is regadenoson. Regadenoson has demonstrated non-inferiority to adenosine for detecting reversible myocardial perfusion defects in phase 3 trials, and patients were more comfortable during the regadenoson stress procedure than during an adenosine infusion. As regadenoson dosing is not dependent on patient weight or renal impairment and can be administered by rapid injection, it has the potential to simplify the stress procedure, reduce costs, and streamline the working day for the staff of the nuclear medicine department. In this review, the need to improve on older pharmacologic stress agents will be considered, along with an assessment of how $A_{2 A}$ receptor agonists fulfill that potential. Practical aspects of regadenoson are reviewed, and the impact that $A_{2 A}$ receptor agonist use may have on the nuclear medicine department is evaluated.

Received Jul. 10, 2009; revision accepted May 10, 2010.

For correspondence or reprints contact: Sara G. Johnson, CNMT, NCT, VA San Diego Healthcare System, Nuclear Medicine Service 115, 3350 La Jolla Village Rd., San Diego, CA 92161.

E-mail: saragjohnson@hotmail.com

COPYRIGHT ( 2010 by the Society of Nuclear Medicine, Inc.
Key Words: myocardial perfusion imaging; regadenoson; $A_{2 A}$ receptor agonists

J Nucl Med Technol 2010; 38:163-171

DOI: 10.2967/jnmt.109.065581

$\mathbf{M}$ yocardial perfusion imaging (MPI) using radionuclides is a well-validated, noninvasive method to aid in the determination of diagnosis and prognosis for patients with suspected or known coronary artery disease. Myocardial blood flow during hyperemia is inversely related to coronary artery stenosis when the degree of stenosis is greater than $40 \%$ (1). Because of the inability of stenotic vessels to dilate, there is a disparity in flow between normal and diseased myocardium (2). Images of relative myocardial perfusion are obtained by PET or SPECT after radionuclide administration. Images obtained during myocardial hyperemia, induced by exercise or pharmacologic stress agents, are compared with images obtained at rest to determine the coronary flow reserve and identify areas of compromised myocardial perfusion.

The preferred modality for inducing hyperemia is physical exercise, because of the additional diagnostic information provided from the exercise tolerance of the patient and the hemodynamic response, heart rate recovery, and electrocardiographic changes during exercise (3). Pharmacologic stress agents are indicated for patients with medical conditions for which exercise stress is not suitable or is unsafe, or for those unable to achieve the cardiac workload necessary for optimal imaging $(4,5)$. Pharmacologic stress MPI may therefore be more commonly used in patients who are elderly, debilitated, or morbidly obese; have musculoskeletal or neurologic limitations; or have severe pulmonary disease, peripheral vascular disease, artificial pacemakers and implantable cardioverter defibrillators, left bundle branch block, or underlying rhythm disturbances (3). Adequate exercise is dependent on the patient's achieving at least $85 \%$ of the age-adjusted maximal predicted heart rate (220 beats per minute minus patient age), a workload of more than 5 metabolic equivalents, and at least $3 \mathrm{~min}$ of exercise to completion of stage 1 of the standard Bruce 
protocol $(6,7)$. An inability to achieve these targets is often the case in patients who are older, have noncardiac physical limitations, take concomitant medications, or lack the necessary motivation (7).

Approximately 8.5 million stress MPI studies are performed yearly in the United States, of which around $40 \%$ use vasodilator pharmacologic stress agents (8). Furthermore, the use of pharmacologic stress agents or a combination of pharmacologic stress and low-level exercise is increasing $(9,10)$. In the future, the number of stress MPI studies is expected to rise as the population ages and the prevalence of cardiovascular risk factors, such as obesity and diabetes, increases.

The aim of this article is to provide an overview of pharmacologic stress agents, focusing on regadenoson, the first $\mathrm{A}_{2 \mathrm{~A}}$ receptor agonist approved by the U.S. Food and Drug Administration for use as a pharmacologic stress agent in MPI. The available data for regadenoson are reviewed, with particular emphasis on the practical aspects that are relevant to nuclear medicine staff. The impact of the use of such $\mathrm{A}_{2 \mathrm{~A}}$-selective agonists on patients, staff, and nuclear medicine departments as a whole is considered.

\section{PHARMACOLOGIC STRESS AGENTS}

Most pharmacologic stress MPI procedures use the vasodilators adenosine or dipyridamole, with the catecholamine dobutamine used when vasodilators are contraindicated. Definitions of common pharmacologic terms used in this article can be found in Table 1 .

Adenosine induces hyperemic coronary flow to the heart by stimulating the $\mathrm{A}_{2 \mathrm{~A}}$ adenosine receptors on the smooth muscle cells of coronary vessels, which causes their dilation (11). Dipyridamole indirectly activates the same pathway by inhibiting the cellular uptake of adenosine and, consequently, increasing its interstitial concentrations. The excess adenosine nonselectively activates all adenosine receptor subtypes, including the $A_{2 \mathrm{~A}}$ receptors, resulting in vasodilation. Because diseased vessels cannot dilate, images of relative myocardial perfusion can identify stenoses.

Up to $80 \%$ of patients experience side effects resulting from the use of adenosine or dipyridamole for pharmacologic stress MPI (12-16). Most of these side effects are mild and include flushing, chest pain, dyspnea, dizziness, and nausea. These frequent side effects, although not serious, are uncomfortable for the patient and create a need for increased monitoring time and care by the nuclear medicine staff. More serious events, including bronchospasm, atrioventricular block, and peripheral vasodilation (resulting in hypotension), are observed with lower frequency $(7,12$, 14-16). Patients with asthma and chronic obstructive pulmonary disease (COPD) are at increased risk of bronchoconstriction and respiratory problems resulting from the use of adenosine or dipyridamole $(7,17,18)$. The use of dipyridamole-containing medications and the adenosine receptor antagonists aminophylline, theophylline, and caffeine are heavily restricted before the procedure (7). The dose of adenosine or dipyridamole is adjusted according to patient weight and administered by infusion over several minutes, with vital signs and side effects monitored during the infusion $(7,9)$. Both agents cause a 4 - to 4.5 -fold increase in coronary blood flow (19), which is higher than ideal because of the nonlinear uptake of the radionuclides at higher myocardial flow rates (20). The half-life of adenosine is extremely short $(<10 \mathrm{~s})$, whereas the half-life of dipyridamole is approximately $30-45 \mathrm{~min}$ (7).

Dobutamine primarily stimulates $\beta_{1}$-adrenergic receptors, causing an increase in heart rate, blood pressure, and myocardial contractility and, therefore, an increased myocardial oxygen demand and consequent hyperemia. Side effects observed during dobutamine stress are similar in frequency to those observed for adenosine and dipyridamole and include palpitations, chest pain, headache, flushing, arrhythmias, and ST-segment depression. Dobutamine stress is contraindicated in patients taking $\beta$-blockers and is not recommended for patients with asthma and COPD (7). The half-life of dobutamine is around 2 min (7).

Adenosine, dipyridamole, and dobutamine all exhibit unfavorable side effect profiles, have common medical and drug contraindications, and have procedures that are inconvenient and challenging for both the patient and the staff, requiring many resources. A theoretic ideal pharmacologic stress agent would induce a 2- to 3-fold increase in coronary blood flow rapidly after administration. This increase would return to baseline soon afterward yet would be maintained long enough to allow the radiopharmaceutical to concentrate adequately in the myocardium and represent the true distribution of ischemia. Additionally, a longer hyperemic response would permit administration by rapid injection, rather than by the constant infusion required for adenosine, dipyridamole, and dobutamine (21). An ideal agent could be used in patients for whom current agents are unsuitable, such as patients who have contraindicated med-

TABLE 1

Definitions of Common Pharmacologic Terms

\begin{tabular}{ll}
\hline \multicolumn{1}{c}{ Term } & D molecule that binds to a receptor and elicits a response by the cell \\
\hline Agonist & $\begin{array}{l}\text { A molecule that binds to a receptor, but rather than causing a response, blocks the binding of an agonist, so } \\
\text { preventing the response }\end{array}$ \\
Antagonist & The tightness with which an agonist/antagonist binds to a receptor (described using the dissociation constant) \\
Affinity & Activity of an agonist against a limited number of receptors \\
Selectivity &
\end{tabular}


ical conditions or cannot safely discontinue any concomitant medications that may interfere with the MPI study. It would also be preferable if the stress agent did not require dose adjustment according to patient weight or renal status. If these ideal characteristics were realized, pharmacologic stress MPI procedures would be better tolerated and simpler for the patient while being faster, easier, and more accurate for nuclear medicine staff. From a cost perspective, fewer ancillary items, such as microbore tubing and large-volume syringes, would be necessary for a rapid injection than for a constant infusion. In addition, there would be no need for infusion pumps, thus reducing the cost and the possibility that an incorrect pump setup might result in dosing errors. If prefilled syringes were available, which is possible only for an agent that does not require dose adjustment, there would also be no dose wastage when procedures are delayed or cancelled. Furthermore, as the U.S. Pharmacopoeia chapter 797 (a federal act that mandates changes in how pharmaceuticals are drawn up, stored, and handled) is implemented at the state level, the practice of nuclear medicine departments performing MPI procedures will be affected. Moving forward, it is likely that agents that facilitate compliance with these higher standards will be favored.

\section{$A_{2 A}$ RECEPTOR AGONISTS}

$A_{2 A}$ receptor stimulation causes the desired coronary vasodilation to induce the myocardial hyperemia required for MPI (11), whereas the nonselective action of adenosine and dipyridamole on the $A_{1}, A_{2 B}$, and $A_{3}$ receptors is deemed predominantly responsible for the side effects associated with these agents $(22,23)$. Of particular interest are the less frequent but more serious adverse events, including bronchospasm (mediated by the $\mathrm{A}_{1}, \mathrm{~A}_{2 \mathrm{~B}}$, and $\mathrm{A}_{3}$ receptors), atrioventricular block ( $\mathrm{A}_{1}$ receptor), and peripheral vasodilation $\left(\mathrm{A}_{2 \mathrm{~B}}\right.$ receptor) resulting in hypotension $(7,12,14$ 18,21-24). More selective $\mathrm{A}_{2 \mathrm{~A}}$ receptor agonists have the potential to mediate the desired level of hyperemia while reducing the frequency of adverse effects (21). Regadenoson, binodenoson, apadenoson, and 2-p-(2-carboxyethyl) phenethylamino-5'- $N$-ethylcarboxamido adenosine (CGS21680) represent a new generation of such $A_{2 A}$ receptor agonists. Regadenoson is, so far, the only agent approved by the Food and Drug Administration for clinical use. Apadenoson and CGS-21680 have been investigated in preclinical trials. To date, there are limited data published for binodenoson (25-30) and even fewer for apadenoson and CGS-21680 (31,32).

In clinical studies, regadenoson and binodenoson appear to provide diagnostic information comparable to that of adenosine while having improved side effect profiles. In a dose-ranging study of binodenoson, side effects were generally milder and were reported less frequently in patients randomized to receive binodenoson than in those who received adenosine, and second- or third-degree atrioventricular block was observed in $3 \%$ of patients who were administered adenosine but in none of the patients who received binodenoson (28). Two randomized, double-blind, crossover phase 3 trials (Vasodilator-Induced Stress In cONcordance with Adenosine (VISION) trials 302 and 305) have been completed with binodenoson (33), and a New Drug Application was submitted to the Food and Drug Administration in December 2008.

As yet, there are no studies comparing regadenoson and binodenoson directly, and the differences between the study designs in the data published so far make comparisons difficult. Initial results indicate that the use of $\mathrm{A}_{2 \mathrm{~A}}$-selective agonists for pharmacologic stress MPI reduces the frequency and severity of side effects for the patient undergoing the procedure.

\section{REGADENOSON}

In vitro studies have demonstrated that regadenoson is a full agonist as a coronary vasodilator and is 10 -fold more potent than adenosine at increasing coronary conductance (34). The selectivity of regadenoson for the $\mathrm{A}_{2 \mathrm{~A}}$ receptor was also demonstrated, with regadenoson having a 13 -fold lower affinity for the $A_{1}$ receptor than for the $A_{2 A}$ receptor and little or no interaction with the $\mathrm{A}_{2 \mathrm{~B}}$ or $\mathrm{A}_{3}$ receptor (34).

As there is a large receptor reserve for $\mathrm{A}_{2 \mathrm{~A}}$-mediated coronary vasodilation, low-affinity agonists have the potential to elicit large-scale, but rapidly terminating, vasodilation with increased specificity for coronary vessels $(34,35)$. It has been shown that the duration of the vasodilatory response is inversely related to the affinity of the agonist to the $A_{2 A}$ receptor (34). Regadenoson has a much lower affinity than binodenoson or CGS-21680 for the $\mathrm{A}_{2 \mathrm{~A}}$ receptor but a higher affinity than adenosine (Fig. 1). In human patients, a rapid dose of regadenoson mediated an increase in coronary blood flow of 2.5-fold or greater for 2-3 min (36). This is close to the theoretic ideal response for an MPI stress agent and enables regadenoson to be administered by a single rapid injection (21). In a study of 16 patients with varying degrees of renal function, it was demonstrated that regadenoson does not require dose adjustment according to the renal function of the patient (37). Although the effective half-life of regadenoson was increased with decreased renal function, the maximum plasma concentrations and the pharmacokinetic parameters associated with distribution showed only minor differences between treatment groups, and the plasma concentration-time profiles were not significantly altered early after dosing (38). Additionally, the regadenoson dose does not need to be adjusted according to patient weight.

Initial studies of regadenoson in humans were positive, demonstrating that a rapid (over $10 \mathrm{~s}$ ) intravenous injection of regadenoson was well tolerated, with no serious side effects observed. However, these initial studies were small and not powered to investigate the clinical efficacy of regadenoson versus adenosine (36-39).

The aim of the Adenoscan Versus Regadenoson Comparative Evaluation for Myocardial Perfusion Imaging 
FIGURE 1. Reversal time of coronary vasodilation in high-affinity and lowaffinity $A_{2 A}$ agonists $(21,34) . \quad K_{i}=$ dissociation constant of inhibitor; $\mathrm{t}_{0.5}=$ time to $50 \%$ reversal if coronary conductance increases in rat isolated perfused hearts. (Reprinted with permission of (34).)

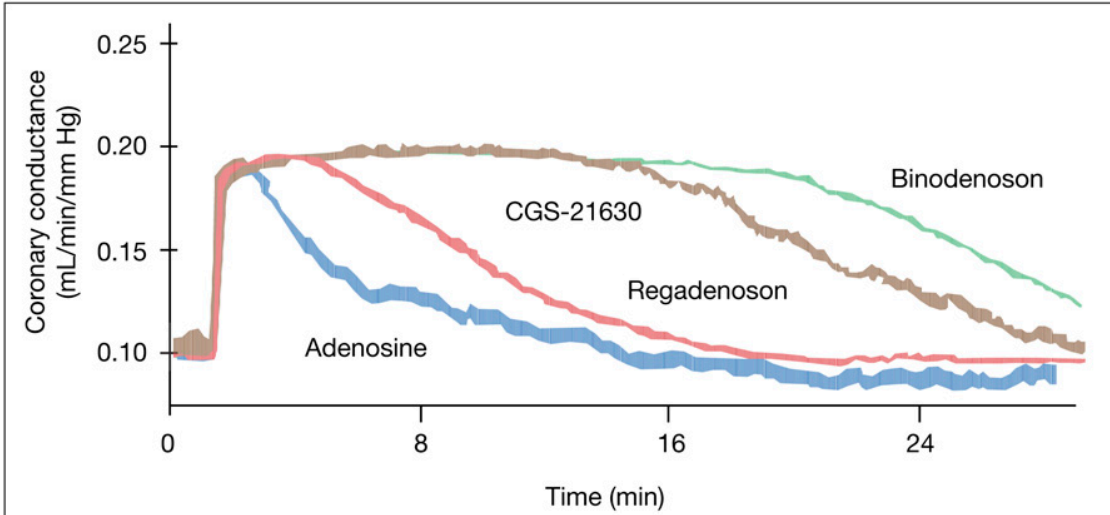

\begin{tabular}{|l|c|c|}
\hline Agonist & $\begin{array}{c}\text { Affinity for } \mathbf{A}_{2 \mathbf{A}} \text { receptor } \\
\left(\mathbf{K}_{\mathbf{i}}, \mathbf{n m o l} / \mathbf{L}\right)\end{array}$ & $\begin{array}{c}\text { Duration of action } \\
\left(\mathbf{t}_{\mathbf{0}} \mathbf{\text { m }} \mathbf{\text { min}} \mathbf{\text { }}\right)\end{array}$ \\
\hline Adenosine & $2,700-5,000$ & $1.6 \pm 0.1$ \\
\hline Regadenoson & 1,095 & $5.2 \pm 0.2$ \\
\hline CGS-21680 & 157 & $14.5 \pm 0.5$ \\
\hline Binodenoson & 21 & $21.9 \pm 0.9$ \\
\hline
\end{tabular}

(ADVANCE MPI) 1 and 2 trials was to demonstrate the non-inferiority of regadenoson, compared with adenosine, by examining the concordance of images detecting reversible myocardial perfusion defects $(40,41)$. ADVANCE MPI 1 and 2 were methodologically identical, double-blind, randomized, active-comparator, double-dummy, multicenter, phase 3 trials. The results of ADVANCE MPI 2 have been published (40), together with a combined analysis of the results of the 2 trials (41).

Patients older than 18 y who had been referred for pharmacologic stress SPECT MPI were eligible for inclusion in the ADVANCE MPI trials (40). All patients first underwent an initial adenosine study and were then randomized 2:1 to a second study using regadenoson or adenosine. In the combined ADVANCE MPI 1 and 2 efficacy population, 1,240 patients received regadenoson and 631 patients received adenosine. All MPI procedures were performed according to the American Society of Nuclear Cardiology guidelines (42). Detailed methods are available in the original publications and are summarized here $(40,41)$. Three expert readers independently scored blinded images, and reversible myocardial perfusion defects were identified and categorized as none to minimal, small to moderate, or large. The overall diagnosis and image quality was also assessed by each reader.

The agreement rates between the initial adenosine procedure and the second randomized procedure with either adenosine or regadenoson were almost identical. The interpretation agreement rate ranged from approximately $50 \%$ in patients with a small to moderate or large extent of ischemia to approximately $85 \%$ in patients with no or minimal ischemia (Fig. 2). Age, sex, body mass index, or diabetes mellitus did not affect the agreement rates (41). Overall, $92 \%$ of images were rated as good or excellent for both agents. Thus, in the ADVANCE MPI trials, regadenoson achieved non-inferiority to adenosine for pharmacologic stress MPI.

Hemodynamic evaluations performed in the ADVANCE MPI trials showed that regadenoson caused a more rapid and greater peak increase in heart rate than did adenosine and that heart rate returned to baseline more slowly in the patients administered regadenoson. Systolic and diastolic blood pressure decreased by a similar extent with both agents, but hemodynamic recovery was more rapid for patients who received adenosine than for those who received regadenoson (40).

Side effects were rated as mild, moderate, or severe. As a measure of tolerability, patients were asked to rate how they felt during the procedure. Additionally, patients were asked

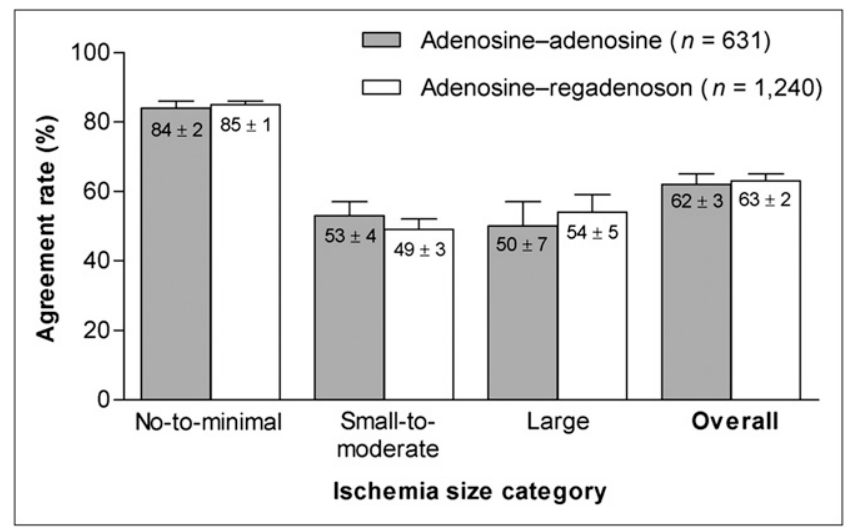

FIGURE 2. Agreement rates of adenosine-adenosine and adenosine-regadenoson stress MPI studies with respect to extent of ischemia in combined ADVANCE MPI 1 and 2 study population (41). Data are median \pm SE. 
to compare the initial study (adenosine) with the second procedure (adenosine or regadenoson). In both the ADVANCE MPI 2 analysis and the combined analysis, patients who received regadenoson had a lower side effect symptom score for the prespecified combination of the most common side effects (chest pain, dyspnea, and flushing), which remained lower for regadenoson when all symptoms were included $(40,41)$. Headache and gastrointestinal discomfort were observed more frequently in the regadenoson group. This complaint has also been observed in postmarketing experience and is discussed in more detail below.

In the combined ADVANCE MPI 1 and 2 populations, first-degree atrioventricular block was observed in $2.8 \%$ ( $n=34$ ) of patients who received regadenoson, compared with $7.0 \%(n=43)$ of those who received adenosine, and second-degree atrioventricular block was observed in $0.1 \%$ $(n=1)$ of patients who received regadenoson, compared with $1.5 \%(n=9)$ of those who received adenosine $(P=$ $0.001)(41)$. There were no instances of third-degree atrioventricular block. However, by study design, patients with a history of high-degree atrioventricular block or those who experienced atrioventricular block during the initial adenosine stress procedure were not randomized to the second procedure. The reduced incidence of atrioventricular block with regadenoson, compared with adenosine, is attributed to the reduced affinity of regadenoson for the $A_{1}$ receptor. Patients who received regadenoson were more comfortable, and when questioned if they preferred the first (adenosine) or second (adenosine or regadenoson) procedure, $62 \%$ of patients randomized to receive regadenoson preferred the second procedure, compared with $43 \%$ of patients who were randomized to receive a second adenosine procedure. The high number of patients who preferred the second adenosine test is attributed to their being more prepared for the procedure and side effects the second time around (40).

These studies demonstrated that regadenoson was noninferior for detecting reversible myocardial perfusion defects while being better tolerated than adenosine. The $\mathrm{A}_{2 \mathrm{~A}}$ specificity of regadenoson does not completely eradicate the side effects that were thought to be solely determined by adenosine receptor types other than $A_{2 A}$. The authors hypothesized that side effects such as chest pain and dyspnea, thought to be the result of $\mathrm{A}_{1}$ receptor stimulation, may be a consequence of sympathetic stimulation (40).

\section{PRACTICAL ASPECTS}

The improved tolerability and reduction in the number of serious adverse events together with similar clinical efficacy, compared with adenosine in the ADVANCE MPI trials, indicates that regadenoson represents an improvement over traditional pharmacologic stress agents.

The Nuclear Medicine Service of the VA San Diego Healthcare System has performed approximately 2,000 MPI procedures using regadenoson since 2008. In our clinical experience, the incidence of gastrointestinal discomfort and persistent headache appears to be higher for regadenoson than for adenosine. In most patients, headaches seem to be alleviated by intake of caffeine after the stress procedure. Nausea and vomiting, although infrequent, may occur shortly after the injection of regadenoson and are usually resolved by 3-4 min after administration. Discussions with other facilities using regadenoson have raised speculation as to the cause of these side effects; some believe that the vomiting may be due to stimulation of $\mathrm{A}_{2 \mathrm{~A}}$ receptors in the stomach, whereas others believe that it may be related to consumption of water before the stress procedure. In some nuclear medicine departments, patients are given water before the acquisition of images at rest to prevent gut activity from interfering with the imaging. If the stress procedure is performed on the same day as the rest scan, the patient's stomach may be full, which may be partly responsible for the nausea and vomiting observed.

These side effects may also be related to how fast the regadenoson dose is administered. Regadenoson should be administered as a rapid injection over approximately $10 \mathrm{~s}$ followed by a $5-\mathrm{mL}$ saline flush. The radiopharmaceutical is injected 10-20 s later, or 25-35 s after the start of the procedure, followed by another saline flush (Fig. 3). We recommend the use of a digital stopwatch to ensure that the injection timing sequence is followed appropriately. When nuclear technologists first use regadenoson, they may tend to rush the regadenoson injection. In our department, after the importance of a steady injection of regadenoson over 8-10 s had been emphasized, and after a sufficient period of practical experience with regadenoson, the staff noticed a reduction in the number of patients with nausea and vomiting. Additionally, the manner in which the regadenoson syringe seal is broken is important. On removal from the package, the syringe must be pulled back and twisted clockwise 3 times to break the seal on the contents before injection. This practice allows the regade-

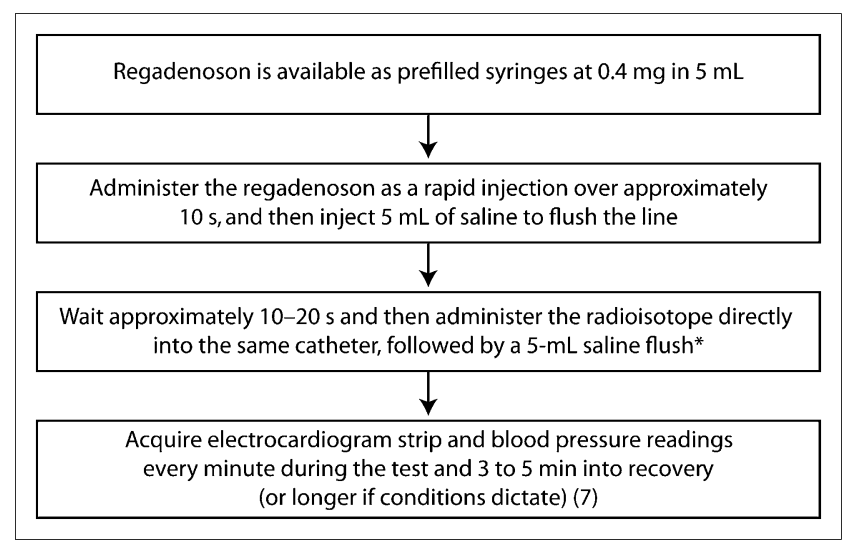

FIGURE 3. Administration of regadenoson. *Aminophylline may be given 1-2 min after injection of isotope to reverse persistent or severe side effects. 
noson to be administered evenly over the recommended 10 -s period. Thus, a rapid administration of regadenoson may be partly responsible for the increased incidence of nausea and vomiting. Further studies are needed to determine the underlying factors causing the increase in these mild side effects. Our primary purpose in mentioning these issues here is to prepare the nuclear medicine technologist to be vigilant for an increased incidence of vomiting and persistent headache, compared with adenosine and dipyridamole, and to highlight some potential causes.

In the event of lingering side effects, aminophylline can be administered after the radiopharmaceutical has been injected and had adequate time to distribute (at least 1-2 min after injection of the radiopharmaceutical). For regadenoson-treated patients in the ADVANCE MPI trials, the most common adverse events for which intervention with aminophylline was considered appropriate were angina pectoris, headache, electrocardiogram changes/ST-segment depression, and chest pain. Aminophylline was infused at doses ranging from 72 to $300 \mathrm{mg}$ after an average of $15 \mathrm{~min}$ from the start of the regadenoson infusion (43).

The pharmacologic stress procedure is faster and less complicated for regadenoson than for other agents. A flow chart of the regadenoson stress procedure is shown in Figure 3. Because regadenoson has a lower affinity for the $A_{2 A}$ receptor than does adenosine (resulting in longer dilation response) and does not require dose adjustment, regadenoson can be administered by a single rapid injection of 0.4 $\mathrm{mg}$ in $5 \mathrm{~mL}(7,34)$. Administering regadenoson by rapid injection has both practical and cost implications. Not only is a rapid injection easier for staff and patients, but regadenoson dosing does not require the costly materials needed for the constant infusion of adenosine or dipyridamole, such as infusion pumps, microbore tubing, and large-volume syringes. Quantifying the exact savings that can be realized is difficult because of the varying costs among different types of institutions, and a detailed cost analysis is beyond the scope of this paper. The response duration of binodenoson means that it likely can also be administered by this method $(27,28)$. However, the length of the binodenoson effect $(>14 \mathrm{~min})$ may require an increase in the monitoring time for each patient.

Additionally, dose adjustment according to patient weight or renal function not only is extra work for the nuclear medicine staff but also increases the possibility of dosing error. A stress agent that does not require dose adjustment can be supplied as prefilled syringes. Any unused agent can then be returned to storage for use in a subsequent procedure, whereas an individually prepared adenosine, dipyridamole, or dobutamine dose would be wasted if a procedure was cancelled or rescheduled. In addition, the use of prefilled syringes facilitates compliance with many of the requirements of U.S. Pharmacopoeia 797. As part of this legislation, doses drawn from vials will have to be used within $1 \mathrm{~h}$, and the syringes that contain these doses will have to be adequately labeled. The increased scrutiny could increase the already high wastage of doses for adenosine and dipyridamole. Thus, the use of prefilled syringes would have a positive effect on the nuclear medicine department by facilitating conformity with U.S. Pharmacopoeia 797 and lowering costs by reducing wastage.

Regadenoson is contraindicated in patients with secondor third-degree atrioventricular block or sinus node dysfunction without a functioning artificial pacemaker and, because of the potential for drug-induced arterial vasodilation, in patients with systolic blood pressure below 90 $\mathrm{mm} \mathrm{Hg} \mathrm{(7).} \mathrm{The} \mathrm{use} \mathrm{of} \mathrm{dipyridamole-containing} \mathrm{medica-}$ tions within $48 \mathrm{~h}$ before the regadenoson procedure, or of aminophylline within $24 \mathrm{~h}$ before the procedure, should be avoided (7). Methylxanthines, including caffeine and theophylline, should be avoided for $12 \mathrm{~h}$ before regadenoson stress (7). Inadvertent caffeine intake is a major cause of cancellations and rescheduling of procedures, an important waste of time and money for the nuclear medicine department. Mounting evidence suggests that regadenoson may allow greater flexibility with regard to caffeine intake before the MPI procedure. In a pilot study of 41 healthy volunteers, Gaemperli et al. (44) investigated the effects of caffeine on myocardial blood flow during regadenoson stress. Patients were randomized to receive $200 \mathrm{mg}$ of caffeine (equivalent to 2 cups of coffee) or placebo before myocardial blood flow was determined at rest $(2 \mathrm{~h}$ after caffeine ingestion) and immediately after intravenous administration of regadenoson $(0.4 \mathrm{mg})$. After a 2 - to 14-d crossover period, the patients who had received the caffeine underwent imaging again with placebo, and the patients who had received placebo underwent imaging with caffeine. The myocardial blood flow after caffeine was similar to that after placebo both at rest $(1.13 \pm 0.04$ and $1.06 \pm$ $0.05 \mathrm{~mL} / \mathrm{min} / \mathrm{g}$, respectively) and during regadenoson stress $(2.98 \pm 0.14$ and $3.05 \pm 0.14 \mathrm{~mL} / \mathrm{min} / \mathrm{g}$, respectively). Similarly, the coronary flow reserve after caffeine was not significantly different from that after placebo $(2.75 \pm$ 0.16 and $2.97 \pm 0.16 \mathrm{~mL} / \mathrm{min} / \mathrm{g}$, respectively). The data from this study demonstrated with 1 -sided $95 \%$ confidence that any coronary flow reserve reduction associated with caffeine intake is less than $20 \%$ for regadenoson stress. The findings of this study indicate that use of regadenoson may potentially reduce the necessity for caffeine restriction before a stress procedure. This possibility is to be addressed further in future trials.

Other pilot studies indicate that regadenoson may be suitable for use in patients with COPD (45) and asthma (46). Such patients are at increased risk of bronchoconstriction and respiratory problems because of the action of adenosine and dipyridamole on the $\mathrm{A}_{2 \mathrm{~B}}$ or $\mathrm{A}_{3}$ receptors, resulting in mast cell degranulation and release of $\mathrm{IgE}$ and histamine $(23,47-50)$. Thus, the $\mathrm{A}_{2 \mathrm{~A}}$ specificity of regadenoson may potentially prevent these reactions and allow use in patients with reactive airways.

Leaker et al. (46) enrolled patients with mild or moderate asthma who were deemed to be particularly at risk of 
bronchoconstriction, having demonstrated bronchial hyperreactivity in a standard adenosine monophosphate challenge test. Subjects were randomized 1:1 to receive a single intravenous $0.4-\mathrm{mg}$ bolus of either regadenoson or placebo followed by a 1-to 14-d washout period, before receiving the other treatment. In total, 47 patients completed the study. No significant difference was observed in the mean forced expiratory volume in $1 \mathrm{~s}\left(\mathrm{FEV}_{1}\right)$ at any time after regadenoson or placebo administration. The mean ratio of the $\mathrm{FEV}_{1}$ values at each posttreatment time point to the baseline values was significantly greater after regadenoson administration than after placebo from 10 to $60 \mathrm{~min}$ after treatment and was numerically higher (although not significantly so) for other time points. The greatest $\mathrm{FEV}_{1}$ reduction $(-36.2 \%)$ was observed after a patient was administered regadenoson, although this reduction was asymptomatic and reversed spontaneously. The incidence of bronchoconstrictive reactions, defined as an $\mathrm{FEV}_{1}$ reduction of more than $15 \%$ from baseline within $2 \mathrm{~h}$ after treatment, was similar after regadenoson $(2 / 47$, or $4.3 \%)$ and placebo $(2 / 48$, or $4.2 \%)$; none of these patients had a serious adverse event or a pulmonary adverse event. More patients experienced an adverse event after receiving regadenoson $(98 \%)$ than after receiving placebo (8\%). After receiving regadenoson, $34 \%$ of patients experienced dyspnea $0-15 \mathrm{~s}$ after dosing. This dyspnea did not correlate with bronchoconstriction in this study, unlike a similar study of adenosine (51), and none of these episodes required treatment.

Thomas et al. (45) enrolled patients with moderate COPD (stage II) and severe COPD (stage III), according to the staging criteria of the scientific committee of the Global Initiative for Chronic Obstructive Lung Disease (52). Patients were randomized 1:1 to receive a single intravenous $0.4-\mathrm{mg}$ dose of regadenoson or placebo, followed by a 7 - to 14-d crossover period, before receiving the alternate treatment. There were no statistically significant differences in the least squares mean $\mathrm{FEV}_{1}$ or forced vital capacity at any time point after regadenoson or placebo administration. The maximum reduction in $\mathrm{FEV}_{1}$ was also similar between regadenoson and placebo: $0.11 \pm 0.02$ and $0.12 \pm 0.02 \mathrm{~L}$, respectively (least squares mean $\pm \mathrm{SE}$ ). New-onset wheezing occurred in $6 \%$ of patients after receiving regadenoson and $12 \%$ of patients after receiving placebo. Bronchoconstrictive reactions (defined as in the asthma study) were observed in $12 \%(6 / 49)$ of patients after receiving regadenoson and 6\% (3/49) of patients after receiving placebo; no treatment was administered for any of these events. Adverse events were experienced by $86 \%$ of patients after regadenoson and $12 \%$ of patients after placebo, with dyspnea the most common adverse event reported after regadenoson administration $(61 \%)$. All incidences of dyspnea resolved spontaneously within $38 \mathrm{~min}$. Dyspnea was not reported by any patients after receiving placebo.

In these trials, no difference was observed in lung function parameters between patients administered regade- noson and patients administered placebo. Regadenoson is not contraindicated in patients with COPD or asthma, although it is recommended that patients discuss their respiratory history and administration of pre- and poststudy bronchodilator therapy before undergoing regadenoson stress MPI (53). Furthermore, it is recommended that patients with ongoing wheezing not undergo regadenoson stress MPI (7). Further research is required to confirm these observations before regadenoson can confidently be used for MPI procedures in these patient populations; a phase 4 study of the safety and tolerance of regadenoson in patients with COPD and asthma is ongoing. Having an agent that is safe for these patient groups would allow a single protocol to be used for most patients, reducing the number of protocols that have to be in place.

Combining pharmacologic stress with low-level exercise for MPI is thought to decrease side effects, improve image quality, and increase test sensitivity $(10,54)$. The use of regadenoson in conjunction with low-level exercise has been investigated (55). In total, 60 patients underwent an initial MPI procedure with adenosine administered in the supine position, before being randomized to a second procedure with either regadenoson in combination with $4 \mathrm{~min}$ of low-level treadmill exercise $(2.7 \mathrm{~km} / \mathrm{h}$ at $0 \%$ grade $)(n=$ 39) or placebo in combination with 4 min of low-level treadmill exercise and radiotracer placebo $(n=21)$. Patients experienced 1 or more adverse events with higher frequency after the adenosine-supine procedure than after the regadenoson-exercise procedure $(95 \%$ vs. $77 \%$, respectively), with severe adverse events (abdominal pain, chest pain, ST-segment depression, neck pain, headache, and paresthesia) observed in $6.7 \%$ of patients during the adenosine-supine procedure and in no patients during the regadenoson-exercise or placebo-exercise procedures. Images obtained using the regadenoson-exercise protocol were judged by all experts to be at least of the same quality as images obtained using the adenosinesupine protocol and were judged to be better by $26 \%(P=$ 0.002) (55). It is thought that exercise induces a sympathetic response that compensates for the hypotension and other adverse events that may result from pharmacologic stress agent administration. A limitation of this study is that the regadenoson-exercise protocol was not compared with an adenosine-exercise protocol. Further study will determine whether this is an optimal procedure for regadenoson stress MPI.

\section{CONCLUSION}

Initial evidence suggests that $\mathrm{A}_{2 \mathrm{~A}}$-selective agonists such as regadenoson are an improvement on currently available pharmacologic stress agents such as adenosine, dipyridamole, and dobutamine. The fact that regadenoson has clinical efficacy comparable to adenosine, and better safety and tolerability, is probably due to the $A_{2 \mathrm{~A}}$ receptor selectivity of regadenoson. As a low-affinity $\mathrm{A}_{2 \mathrm{~A}}$ agonist, regadenoson has an extent and duration of hyperemic response that is 
close to ideal for MPI and allows administration by rapid injection. The lack of necessity for dose adjustment according to patient weight and renal function allows regadenoson to be supplied in prefilled syringes; this is important to reduce waste and facilitate compliance with legislation. The less complex regadenoson administration will simplify the work of the nuclear medicine staff and offer patients a quicker and easier procedure with potentially milder side effects. In turn, patient satisfaction, department efficiency, and cost-effectiveness should improve.

Further research will reveal if regadenoson can be used in patients with reactive airway disease and how efficacy is affected by caffeine and other drugs normally contraindicated for pharmacologic stress procedures. A reduction of preprocedural requirements would be an improvement, as patient preparation for the procedure would be simplified and the number of cancelled and rescheduled procedures, and the associated costs, would be reduced.

Regadenoson appears to have many of the attributes of an ideal pharmacologic stress agent for MPI. Further research will reveal if other $\mathrm{A}_{2 \mathrm{~A}}$ agonists have similar clinical utility.

\section{ACKNOWLEDGMENTS}

Editorial support was provided by Emily Hutchinson and Elaine Griffin, medical writers at Envision Scientific Solutions funded by Astellas Pharma Global Development, Inc.

\section{REFERENCES}

1. Uren NG, Melin JA, De Bruyne B, Wijns W, Baudhuin T, Camici PG. Relation between myocardial blood flow and the severity of coronary-artery stenosis. $N$ Engl J Med. 1994;330:1782-1788.

2. Iskandrian AS, Verani MS, Heo J. Pharmacologic stress testing: mechanism of action, hemodynamic responses, and results in detection of coronary artery disease. J Nucl Cardiol. 1994;1:94-111.

3. Boger LA, Volker LL, Hertenstein GK, Bateman TM. Best patient preparation before and during radionuclide myocardial perfusion imaging studies. $\mathrm{J} \mathrm{Nucl}$ Cardiol. 2006;13:98-110.

4. Verzijlbergen JF, Vermeersch PH, Laarman GJ, Ascoop CA. Inadequate exercise leads to suboptimal imaging: thallium-201 myocardial perfusion imaging after dipyridamole combined with low-level exercise unmasks ischemia in symptomatic patients with non-diagnostic thallium-201 scans who exercise submaximally. J Nucl Med. 1991;32:2071-2078.

5. Iskandrian AS, Heo J, Kong B, Lyons E. Effect of exercise level on the ability of thallium-201 tomographic imaging in detecting coronary artery disease: analysis of 461 patients. J Am Coll Cardiol. 1989;14:1477-1486.

6. Patel RN, Arteaga RB, Mandawat MK, Thornton JW, Robinson VJ. Pharmacologic stress myocardial perfusion imaging. South Med J. 2007;100: 1006-1014.

7. American Society of Nuclear Cardiology Web site. ASNC Imaging Guidelines for Nuclear Cardiology Procedures. Available at: http://www.asnc.org/imageup loads/ImagingGuidelinesStressProtocols021109.pdf. Accessed July 8, 2010.

8. Iskandrian AE. A new generation of coronary vasodilators in stress perfusion imaging. Am J Cardiol. 2007;99:1619-1620.

9. Miyamoto MI, Vernotico SL, Majmundar H, Thomas GS. Pharmacologic stress myocardial perfusion imaging: a practical approach. J Nucl Cardiol. 2007; $14: 250-255$

10. Thomas GS, Prill NV, Majmundar H, et al. Treadmill exercise during adenosine infusion is safe, results in fewer adverse reactions, and improves myocardial perfusion image quality. J Nucl Cardiol. 2000;7:439-446.
11. Belardinelli L, Shryock JC, Snowdy S, et al. The $\mathrm{A}_{2 \mathrm{~A}}$ adenosine receptor mediates coronary vasodilation. J Pharmacol Exp Ther. 1998;284:1066-1073.

12. Cerqueira MD, Verani MS, Schwaiger M, Heo J, Iskandrian AS. Safety profile of adenosine stress perfusion imaging: results from the Adenoscan Multicenter Trial Registry. J Am Coll Cardiol. 1994;23:384-389.

13. Hendel RC, Jamil T, Glover DK. Pharmacologic stress testing: new methods and new agents. J Nucl Cardiol. 2003;10:197-204.

14. Ranhosky A, Kempthorne-Rawson J. The safety of intravenous dipyridamole thallium myocardial perfusion imaging. Intravenous Dipyridamole Thallium Imaging Study Group. Circulation. 1990;81:1205-1209.

15. Lette J, Tatum JL, Fraser S, et al. Safety of dipyridamole testing in 73,806 patients: the Multicenter Dipyridamole Safety Study. J Nucl Cardiol. 1995; 2:3-17.

16. Mohiuddin SM, Esterbrooks DJ, Gupta NC, Hilleman DE. Safety of different dosages of intravenous adenosine used in conjunction with diagnostic myocardial imaging techniques. Pharmacotherapy. 1993;13:476-480.

17. Burkhart KK. Respiratory failure following adenosine administration. Am J Emerg Med. 1993;11:249-250.

18. Drake I, Routledge PA, Richards R. Bronchospasm induced by intravenous adenosine. Hum Exp Toxicol. 1994;13:263-265.

19. Chan SY, Brunken RC, Czernin J, et al. Comparison of maximal myocardial blood flow during adenosine infusion with that of intravenous dipyridamole in normal men. J Am Coll Cardiol. 1992;20:979-985.

20. Cerqueira MD. Advances in pharmacologic agents in imaging: new $\mathrm{A}_{2 \mathrm{~A}}$ receptor agonists. Curr Cardiol Rep. 2006;8:119-122.

21. Cerqueira MD. The future of pharmacologic stress: selective $\mathrm{A}_{2 \mathrm{~A}}$ adenosine receptor agonists. Am J Cardiol. 2004;94:33D-40D.

22. Fredholm BB, IJzerman AP, Jacobson KA, Klotz KN, Linden J. International Union of Pharmacology. XXV. Nomenclature and classification of adenosine receptors. Pharmacol Rev. 2001;53:527-552.

23. Shryock JC, Belardinelli L. Adenosine and adenosine receptors in the cardiovascular system: biochemistry, physiology, and pharmacology. Am J Cardiol. 1997;79:2-10.

24. Belardinelli L, Linden J, Berne RM. The cardiac effects of adenosine. Prog Cardiovasc Dis. 1989;32:73-97.

25. Barrett RJ, Lamson MJ, Johnson J, Smith WB. Pharmacokinetics and safety of binodenoson after intravenous dose escalation in healthy volunteers. $\mathrm{J} \mathrm{Nucl}$ Cardiol. 2005;12:166-171.

26. Glover DK, Ruiz M, Yang JY, et al. Pharmacological stress thallium scintigraphy with 2-cyclohexylmethylidenehydrazinoadenosine (WRC-0470): a novel, shortacting adenosine $\mathrm{A}_{2 \mathrm{~A}}$ receptor agonist. Circulation. 1996;94:1726-1732.

27. Hodgson JM, Dib N, Kern MJ, Bach RG, Barrett RJ. Coronary circulation responses to binodenoson, a selective adenosine $\mathrm{A}_{2 \mathrm{~A}}$ receptor agonist. $\mathrm{Am} \mathrm{J}$ Cardiol. 2007;99:1507-1512.

28. Udelson JE, Heller GV, Wackers FJ, et al. Randomized, controlled dose-ranging study of the selective adenosine $\mathrm{A}_{2 \mathrm{~A}}$ receptor agonist binodenoson for pharmacological stress as an adjunct to myocardial perfusion imaging. Circulation. 2004;109:457-464.

29. Martin PL, Barrett RJ, Linden J, Abraham WM. Pharmacology of 2-cyclohexylmethylidenehydrazinoadenosine (WRC-0470), a novel, shortacting adenosine $\mathrm{A}_{2 \mathrm{~A}}$ receptor agonist that produces selective coronary vasodilation. Drug Dev Res. 1997;40:313-324.

30. Martin PL, Barrett RJ, Sykes A, Droppleman DA, Wright KF, Mossem $D$. Pharmacology and toxicology of the $\mathrm{A}_{2 \mathrm{~A}}$-adenosine receptor agonist 2-[(cyclohexylmethylene)hydrazino]adenosine (MRE-0470) in the rat. Drug Dev Res. 1997;42:76-85.

31. Glover DK, Ruiz M, Takehana K, et al. Pharmacological stress myocardial perfusion imaging with the potent and selective $\mathrm{A}_{2 \mathrm{~A}}$ adenosine receptor agonists ATL193 and ATL146e administered by either intravenous infusion or bolus injection. Circulation. 2001;104:1181-1187.

32. He ZX, Cwajg E, Hwang W, et al. Myocardial blood flow and myocardial uptake of ${ }^{201} \mathrm{Tl}$ and ${ }^{99 \mathrm{~m}} \mathrm{Tc}$-sestamibi during coronary vasodilation induced by CGS-21680, a selective adenosine $\mathrm{A}_{2 \mathrm{~A}}$ receptor agonist. Circulation. 2000;102:438-444.

33. Udelson JE, Iteld B, Weiland FL, et al. Efficacy and safety of the selective adenosine $\mathrm{A}_{2 \mathrm{~A}}$ receptor agonist binodenoson for pharmacologic stress: results of a phase 3, randomized, double-blind, risk-stratified, active-controlled, crossover trial. Presented at: 57th Annual Scientific Session of the American College of Cardiology; Chicago, Illinois; March 29-April 1, 2008. Latebreaking abstract 409-05.

34. Gao Z, Li Z, Baker SP, et al. Novel short-acting $\mathrm{A}_{2 \mathrm{~A}}$ adenosine receptor agonists for coronary vasodilation: inverse relationship between affinity and duration of action of $\mathrm{A}_{2 \mathrm{~A}}$ agonists. J Pharmacol Exp Ther. 2001;298:209-218.

35. Shryock JC, Snowdy S, Baraldi PG, et al. $\mathrm{A}_{2 \mathrm{~A}}$-adenosine receptor reserve for coronary vasodilation. Circulation. 1998;98:711-718. 
36. Lieu HD, Shryock JC, von Mering GO, et al. Regadenoson, a selective $A_{2 A}$ adenosine receptor agonist, causes dose-dependent increases in coronary blood flow velocity in humans. J Nucl Cardiol. 2007;14:514-520.

37. Gordi T, Blackburn B, Lieu H. Regadenoson pharmacokinetics and tolerability in subjects with impaired renal function. J Clin Pharmacol. 2007;47:825-833.

38. Gordi T, Frohna P, Sun HL, Wolff A, Belardinelli L, Lieu H. A population pharmacokinetic/pharmacodynamic analysis of regadenoson, an adenosine $\mathrm{A}_{2 \mathrm{~A}}$-receptor agonist, in healthy male volunteers. Clin Pharmacokinet. 2006; 45:1201-1212.

39. Hendel RC, Bateman TM, Cerqueira MD, et al. Initial clinical experience with regadenoson, a novel selective A2A agonist for pharmacologic stress singlephoton emission computed tomography myocardial perfusion imaging. $J \mathrm{Am}$ Coll Cardiol. 2005;46:2069-2075.

40. Iskandrian AE, Bateman TM, Belardinelli L, et al. Adenosine versus regadenoson comparative evaluation in myocardial perfusion imaging: results of the ADVANCE phase 3 multicenter international trial. J Nucl Cardiol. 2007; 14:645-658.

41. Cerqueira MD, Nguyen $P$, Staehr P, et al. Effects of age, gender, obesity, and diabetes on the efficacy and safety of the selective $\mathrm{A}_{2 \mathrm{~A}}$ agonist regadenoson versus adenosine in myocardial perfusion imaging. $J$ Am Coll Cardiol Imaging. 2008;1:307-316.

42. Imaging guidelines for nuclear cardiology procedures, part 2. American Society of Nuclear Cardiology. J Nucl Cardiol. 1999;6:G47-G84.

43. Cerqueira MD, Mahmarian JJ, Kinser C, Papatheofanis FJ, Segreti A. Frequency of aminophylline use to attenuate adverse reactions to regadenoson and adenosine [abstract]. J Nucl Cardiol. 2009;16(suppl 4):658.

44. Gaemperli O, Schepis $T$, Koepfli $P$, et al. Interaction of caffeine with regadenoson-induced hyperemic myocardial blood flow as measured by positron emission tomography: a randomized, double-blind, placebo-controlled crossover trial. J Am Coll Cardiol. 2008;51:328-329.

45. Thomas GS, Tammelin BR, Schiffman GL, et al. Safety of regadenoson, a selective adenosine $\mathrm{A}_{2 \mathrm{~A}}$ agonist, in patients with chronic obstructive pulmonary disease: a randomized, double-blind, placebo-controlled trial (RegCOPD trial). J Nucl Cardiol. 2008;15:319-328.

46. Leaker BR, O'Connor B, Hansel TT, et al. Safety of regadenoson, an adenosine $\mathrm{A}_{2 \mathrm{~A}}$ receptor agonist for myocardial perfusion imaging, in mild asthma and moderate asthma patients: a randomized, double-blind, placebo-controlled trial. J Nucl Cardiol. 2008;15:329-336.

47. Fan M, Qin W, Mustafa SJ. Characterization of adenosine receptor(s) involved in adenosine-induced bronchoconstriction in an allergic mouse model. Am J Physiol Lung Cell Mol Physiol. 2003;284:L1012-L1019.

48. Feoktistov I, Biaggioni I. Role of adenosine in asthma. Drug Dev Res. 1996; 39:333-336.

49. Feoktistov I, Polosa R, Holgate ST, Biaggioni I. Adenosine $A_{2 B}$ receptors: a novel therapeutic target in asthma? Trends Pharmacol Sci. 1998;19:148-153.

50. Ryzhov S, Goldstein AE, Matafonov A, Zeng D, Biaggioni I, Feoktistov I. Adenosine-activated mast cells induce IgE synthesis by B lymphocytes: an $\mathrm{A}_{2 \mathrm{~B}}$-mediated process involving Th2 cytokines IL-4 and IL-13 with implications for asthma. J Immunol. 2004;172:7726-7733.

51. Balan KK, Critchley M. Is the dyspnea during adenosine cardiac stress test caused by bronchospasm? Am Heart J. 2001;142:142-145.

52. Fabbri L, Pauwels RA, Hurd SS. Global strategy for the diagnosis, management, and prevention of chronic obstructive pulmonary disease: GOLD Executive Summary updated 2003. COPD. 2004;1:105-141.

53. Astellas Pharma Global Development, Inc. Lexiscan prescribing information. Available at: http://www.astellas.us/docs/lexiscan.pdf. Accessed June 30, 2010 .

54. Thomas GS, Miyamoto MI. Should simultaneous exercise become the standard for adenosine myocardial perfusion imaging? Am J Cardiol. 2004; 94:3D-10D

55. Thomas GS, Thompson RC, Miyamoto MI, et al. The RegEx trial: a randomized, double-blind, placebo- and active-controlled pilot study combining regadenoson, a selective $\mathrm{A}_{2 \mathrm{~A}}$ adenosine agonist, with low-level exercise, in patients undergoing myocardial perfusion imaging. J Nucl Cardiol. 2009;16:63-72. 